Revue belge de géographie

\title{
Trappes de développement et influence de la Politique de Cohésion de l'Union européenne : une exploration géospatiale
}

Development traps and influence of the EU Cohesion Policy: an explorative spatial analysis

\section{Sébastien Bourdin}

\section{(2) OpenEdition}

\section{Journals}

\section{Édition électronique}

URL : http://journals.openedition.org/belgeo/29106

DOI : 10.4000/belgeo.29106

ISSN : 2294-9135

Éditeur :

National Committee of Geography of Belgium, Société Royale Belge de Géographie

\section{Référence électronique}

Sébastien Bourdin, «Trappes de développement et influence de la Politique de Cohésion de l'Union européenne : une exploration géospatiale », Belgeo [En ligne], 2 | 2019, mis en ligne le 30 novembre 2018, consulté le 08 juillet 2020. URL : http://journals.openedition.org/belgeo/29106 ; DOI : https:// doi.org/10.4000/belgeo.29106

Ce document a été généré automatiquement le 8 juillet 2020.

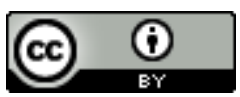

Belgeo est mis à disposition selon les termes de la licence Creative Commons Attribution 4.0 International. 


\section{Trappes de développement et influence de la Politique de Cohésion de l'Union européenne : une exploration géospatiale}

Development traps and influence of the EU Cohesion Policy: an explorative spatial analysis

Sébastien Bourdin

Classification JEL : C21, C26, R58

\section{Introduction}

1 La Politique de Cohésion de l'Union européenne (UE) - instrument financier visant à réduire les inégalités régionales en stimulant à la fois l'investissement pour la croissance et l'emploi, et la coopération territoriale européenne (Commission européenne, 2011) - a fait l'objet de nombreuses études d'évaluation d'impacts de programmes spécifiques, sur différents thèmes, à différentes échelles et sur plusieurs périodes de programmation. Ces types d'études ont souvent utilisé des méthodes qualitatives et/ou quantitatives apportant des évaluations plus ou moins détaillées de cette politique publique européenne (Baslé, 2006 ; Bachtler, Wren, 2006; Ward, Wolleb, 2006; Commission européenne, 2010; Mohl, 2016). D’une manière générale, il faut retenir de ces analyses que la Politique de Cohésion a fait des progrès pour atteindre ses objectifs d'accompagnement du développement économique local et de réduction des inégalités régionales. Dans le même temps, ces évaluations pointent bien souvent une hétérogénéité des résultats en fonction des régions, des périodes et des politiques du fait que les projets financés peuvent être altérés par (i) des délais, (ii) un manque de définition des objectifs ou des buts surdimensionnés, (iii) une insuffisance de coordination et d'aménagement, (iv) des attentes surréalistes ou encore (v) une 
insuffisance de l'accompagnement. On retrouve ainsi dans l'UE des espaces au sein desquels les Fonds européens semblent avoir du mal à atteindre leurs objectifs en termes de développement malgré l'intensité des financements qui y sont concentrés. Par ailleurs, de nombreux chercheurs (Quah, 1996 ; Carrington, 2003 ; Dall'erba, 2005 ; Ertur et al., 2006; Ramajo et al., 2008 ; Mohl, Hagen, 2010 ; Bouayad-Agha et al., 2013 ; Maynou et al., 2016; Percoco, 2017) ont montré que l'efficacité des Fonds structurels européens dépendait largement de l'environnement régional de l'unité géographique traitée par la Politique de Cohésion (localisation relative) et que, par ailleurs, il existait des externalités spatiales liées à l'implémentation des Fonds européens (Dall'erba, Le Gallo, 2008; Dall'erba, Fang, 2017). Autrement dit, il existe des effets de dépendance spatiale entre les régions (Koch, 2004 ; Ertur, Koch, 2006 ; Bourdin, 2010) qui expliquent en partie l'hétérogénéité spatiale de l'impact de cette politique publique européenne de développement territorial (Bourdin, 2018). Ces effets de dépendance spatiale font directement référence au problème d'autocorrélation spatiale (Le Gallo, 2002), autrement dit à la coïncidence de la similarité de valeurs avec la similarité de localisation (Anselin, 2000).

2 C'est dans ce cadre que nous souhaitons interroger la question des liens entre la distribution des Fonds européens et le développement économique régional en prenant en compte les effets de dépendance spatiale (effets de débordement géographique et autocorrélation spatiale), à l'aide d'une méthode exploratoire en statistiques spatiales qui, à ce jour, n'a pas encore été utilisée sur ces questions. L'étude des relations spatiales entre les entités géographiques fait l'objet depuis plusieurs décennies d'un grand intérêt pour les géographes. Parmi les thématiques de recherche sur ce sujet on retrouve l'analyse de l'autocorrélation spatiale qui mesure l'association entre des objets du même type (Cliff, Ord, 1973). Les mesures existantes comprennent des statistiques globales (Moran, 1950 ; Geary, 1954) et locales (Anselin, 1995 ; Getis, Ord, 1992) avec de larges applications telles que l'analyse de la criminalité (ie. Getis, 2010 ; Messner et al., 1999), la démographie (Oliveau, 2010) ou encore les inégalités régionales (Ertur et Koch, 2005; Bourdin, 2010). Un autre axe de recherche se concentre sur la corrélation spatiale ou co-localisation, qui mesure la relation spatiale entre les objets de différents types (Leslie, Kronenfeld, 2011). Ainsi, nous souhaitons utiliser la méthode des indicateurs locaux d'association spatiale bivariés (Anselin et al., 2000) pour identifier de manière paramétrique les concentrations spatiales des covariations spatiales entre la croissance régionale et différentes variables explicatives (dont les Fonds de la Politique de Cohésion) de celle-ci. Grâce à cette méthode, il est ainsi possible de pouvoir identifier des trappes de développement où on peut faire l'hypothèse que l'utilisation de l'argent de la Politique de Cohésion n'a pas eu les effets escomptés, ou au contraire, des regroupements spatiaux de régions enregistrant une croissance importante, grâce en partie aux Fonds européens dépensés. Puisqu'il s'agit d'une méthode exploratoire, l'objectif n'est pas de détecter des relations causales entre les facteurs explicatifs de la croissance régionale (ce que l'économétrie spatiale permet de faire), mais plutôt d'identifier notamment des concentrations spatiales de régions cumulant des difficultés (les trappes de développement), et qui nécessiteraient donc une plus grande attention en termes de politiques publiques. Ceci est particulièrement important dans un contexte de réflexion sur la conception et la mise en œuvre de la future politique régionale post-2020 pour davantage cibler les interventions. 
3 Notre article est structuré comme suit: d'abord nous revenons sur les effets de polarisation spatiale dans l'Union européenne et du rôle de la Politique de Cohésion pour réduire ces inégalités territoriales, puis nous présentons notre méthodologie et enfin terminons par présenter les résultats avant de conclure en ouvrant une discussion sur la mise en œuvre de la Politique de Cohésion post-2020.

\section{Le rôle des Fonds européens pour rompre avec la causation circulaire cumulative de la pauvreté}

\section{De la nécessité d'une Politique de Cohésion pour sortir de la concentration spatiale de la richesse européenne}

Déjà dans les années cinquante, Myrdal (1957) considérait la croissance comme un processus spatial cumulatif susceptible d'accroître les disparités régionales et conduisant à une polarisation spatiale de l'économie. Selon lui, les effets des économies d'agglomération conduisent à un processus cumulatif de croissance créant des différences entre régions et engendrant un cercle vicieux. Il note qu'il existe une «causation circulaire cumulative» de la pauvreté : nulle force équilibrante ne vient corriger les déséquilibres économiques et sociaux. D'autres travaux académiques (Perroux, 1955 ; Williamson, 1965 ; Kaldor 1970 ; Krugman, 1991 ; Krugman, Venables, 1996) arrivent à des conclusions similaires quant à l'existence d'un processus de divergence.

5 L'incidence de la répartition inégale des activités économiques dans l'espace sur la croissance économique des territoires a était mise en évidence au sein notamment du courant théorique dit de la synthèse géographie - croissance (Baumont, 1998). Plusieurs études ont mis en lumière le fait que les disparités régionales ne sont pas réparties de manière aléatoire dans l'espace et que l'environnement géographique d'une région joue un rôle prépondérant dans son développement. La répartition géographique des disparités économiques européennes, étudiée par plusieurs auteurs (López-Bazo et al., 1999 ; Le Gallo, Ertur, 2003 ; Ertur, Koch, 2006 ; Bourdin, 2010) montre une polarisation permanente entre les régions riches du Nord et les régions pauvres $\mathrm{du}$ Sud et de l'Est. Ce constat peut être expliqué notamment par les nouvelles théories de la géographie économique (Krugman 1991; Fujita, 1999) qui montrent que les localisations des activités économiques sont spatialement structurées par certains processus agglomératifs et cumulatifs. En conséquence, nous pouvons dire que la répartition géographique des activités économiques à forte (respectivement faible) valeur ajoutée est spatialement dépendante et tend à faire preuve de persistance dans le temps. De plus, selon cette théorie de la géographie économique, l'environnement économique d'une région semble influencer les perspectives de développement territorial de celle-ci : une région pauvre (respectivement riche) entourée de régions pauvres (respectivement riches) restera dans cet état de développement économique, alors qu'une région pauvre entourée de régions riches a plus de chances d'atteindre un plus haut niveau de développement économique.

Dès lors, pour accélérer les processus de rattrapage, en particulier dans certaines zones d'Europe où il existe une concentration spatiale de régions en retard de développement, il semble nécessaire de mettre en place des politiques publiques de développement territorial. Ceci est d'autant plus justifié que Capello (2007) démontre la 
capacité limitée des processus d'ajustement spontané à rééquilibrer les conditions de départ régionales inégales qui profitent aux régions les plus développées. Comme le soulignait déjà Camagni en 2002, le principe smithien d'avantage absolu est à l'origine d'une compétitivité accrue, engendrant une hiérarchisation de la croissance régionale, augmentant par conséquent les inégalités régionales (Aristei, Perugini, 2012). Dès lors, on voit l'importance des Fonds structurels européens pour rééquilibrer les inégalités et améliorer la cohésion économique, sociale et territoriale de l'UE.

Les Fonds structurels et d'investissement européens (ESIF) sont l'un des instruments de politique économique supranationaux les plus importants de l'UE. Ils visent à réduire les disparités entre les niveaux de développement des différentes régions, tels qu'ils sont codifiés à l'article 174 du Traité sur le fonctionnement de l'UE. Au cours des dernières décennies, l'importance budgétaire de l'ESIF dans le cadre de la construction de politique régionale de l'UE a progressivement augmenté. Dans le cadre financier actuel 2014-2020 pour la première fois dans l'histoire de l'UE, la part la plus importante du budget européen - 351,8 sur 960 milliards d'euros - a été attribuée à cette politique dont 179 milliards d'euros pour les régions dites « les moins développées » auquel il faut ajouter, pour certaines, les Fonds de Cohésion à hauteur de 63 milliards d'euros (source : DG Regio).

8 Compte tenu de la persistance des inégalités économiques entre les régions et les pays de l'UE et le rythme décevant de la reprise économique après la dernière crise économique, un vif débat a vu le jour entre les « eurocrates » et les chercheurs au sujet du réel impact du financement de l'UE sur la croissance régionale. Dès lors, la question de la solidarité entre les Etats-membres et les régions est plus que jamais d'actualité (Bourdin, 2014). La nature distincte des différents programmes de financement a rendu difficile l'identification de leurs effets causaux sur la croissance. De plus, l'éventail des approches consacrées à l'analyse empirique des effets des Fonds structurels a abouti à des conclusions divergentes sur les impacts réels des ESIF. Or, les promoteurs de ces instruments financiers accordent beaucoup d'espoir dans leur potentiel pour accroître le niveau de développement des régions les plus en retard comparé à celui des régions les plus développées.

\section{Une efficacité de la Politique de Cohésion concentrée spatialement}

On recense aujourd'hui une soixante-dizaine d'articles analysant l'impact de la politique régionale européenne mais il n'existe pas de consensus sur la question ${ }^{1}$. Les méthodologies utilisées sont variées allant de modèles dynamiques input-output à des approches contrefactuelles en passant par l'économétrie spatiale. Les résultats des études empiriques évaluant l'efficacité des Fonds européens dans le processus de rattrapage régional sont néanmoins contradictoires et dépendent bien souvent des méthodes utilisées, de la temporalité de l'étude et du choix du maillage territorial (Dall'Erba, Fang, 2017). Ceci amène Molle (2007) à dire que l'efficacité de la politique régionale européenne doit être considérée en termes de plausibilité plutôt que de preuves. 


\section{Quels facteurs expliquent la non-linéarité spatiale de l'impact des Fonds structurels européens sur la croissance régionale?}

10 L'hypothèse de base dans un cadre théorique néoclassique serait que les Fonds structurels favoriseraient la croissance économique en augmentant le taux d'investissement. Dans le même temps, la littérature a montré une hétérogénéité spatiale de l'impact de la Politique de Cohésion sur la croissance régionale qui peut s'expliquer notamment par la théorie de la nouvelle économie géographique. D'une part, de nombreuses études concluent à un effet positif et significatif des ESIF sur la croissance régionale. Sans être exhaustif, on notera certaines contributions récentes (Becker et al., 2012 ; Bouayad-Agha et al., 2013 ; Pelligrini et al., 2013 ; Crescenzi, Giua, 2016; Gagliardi, Percoco, 2017) qui concluent à un impact de la politique régionale sur la croissance. Becker et al. (2012) expliquent cependant que l'allocation de Fonds structurels dans les régions les plus en difficultés des pays riches de l'UE avait des effets plus importants. Gagliardi et Percoco (2017) soulignent le fait que les impacts des Fonds européens sont plus importants dans les régions rurales situées à proximité de grandes agglomération urbaines. Pelligrini et al. (2013) et Crescenzi et Giua (2016) ont comparé les effets de la politique régionale sur le développement économique des territoires ayant bénéficié de ces Fonds (en dessous du seuil des $75 \%$ de la moyenne du PIB/hab. européen) comparés à celles qui en n'ont pas reçu (régions non traitées - au-dessus de ce seuil des $75 \%$ ) et ont conclu à un impact positif. Enfin, les résultats des travaux de Bouayad-Agha et al., (2013) laissent supposer que les actions en faveur de l'objectif 1 ont un effet immédiat sur les taux de croissance, à la différence des fonds structurels globaux. Lorsque l'aspect spatial des données est pris en compte, l'impact des Fonds structurels s'avère encore significatif bien que moins important. D'autre part, d'autres études révèlent que les Fonds européens auraient au contraire un effet nul voire négatif sur la croissance économique des régions telles que celles Dall'Erba et Le Gallo (2007) ou encore de Dall'Erba et al. (2009), en expliquant que la forte autocorrélation spatiale de la croissance régionale - la croissance d'une région est largement influencée par la croissance des régions qui l'entourent - pouvait expliquer des résultats en demi-teinte. Rodríguez-Pose et Fratesi (2004) concluent également que l'influence de l'objectif 1 dans le processus de convergence ne semble pas démontrée. Tout comme Dall'Erba et al. (2009), ils argumentent que l'éducation et l'investissement dans le capital humain constituent un volet important d'une politique de développement régional plus efficace. Un autre facteur explicatif de l'hétérogénéité de l'impact des ESIF sur la croissance s'explique par la gouvernance et le rôle des institutions sur la performance régionale (Glaeser et al., 2004; Ederveen et al., 2006; Arbia et al., 2010; Farole et al., 2011 ; Rodríguez-Pose et Garcilazo, 2015). Les chercheurs expliquent que pendant trop longtemps cette problématique a été oubliée dans la mise en œuvre de la politique régionale. Ils démontrent l'intérêt d'améliorer la qualité de la gouvernance et de réduire l'hétérogénéité institutionnelle (Charron et al., 2015) pour améliorer l'efficacité des Fonds.

11 En somme, les résultats des travaux sur le sujet montrent que l'efficacité de cette politique dépend (i) de la concentration des fonds sur certains thèmes, (ii) des caractéristiques régionales locales/du capital territorial de la région mais aussi (iii) de l'environnement régional (effets de débordement spatiaux). 


\section{Quels impacts des effets spatiaux sur l'efficacité de la Politique de Cohésion ?} publique européenne, l'hétérogénéité de l'impact de la politique trouve ses racines dans des questions liées à la prise en compte des effets de débordement spatiaux et de la performance économique des régions voisines. Par exemple, Le Gallo et al. (2011) expliquent que le développement économique d'une région était largement influencé par celui de ses voisines et que l'impact local des Fonds européens pouvait varier positivement ou négativement au sein de l'Union européenne en conséquence. Dès 1996, Quah soulevait déjà que l'omission de la dimension spatiale du processus de convergence pouvait biaiser les résultats. Il affirmait que la localisation géographique et les effets de débordement géographique importaient plus que les facteurs macroéconomiques nationaux. Ses résultats soulignaient l'importance des retombées spatiales dans la compréhension de la dynamique régionale de distribution de la richesse. Dès lors, les interactions spatiales dues aux retombées géographiques doivent être prises en compte, en particulier lorsque l'on étudie l'efficacité de la Politique de Cohésion. C'est ce qu'ont montré plusieurs chercheurs en soulignant le rôle de l'environnement régional d'une part (Carrington, 2003; Dall'erba, 2005; Ertur et al., 2006 ; Ramajo et al., 2008 ; Mohl, Hagen, 2010 ; Bouayad-Agha et al., 2013 ; Maynou et al., 2016 ; Percoco, 2017) et des externalités spatiales d'autre part (Dall'erba, Le Gallo, 2008 ; Dall'erba, Fang, 2017).

Dans ce cadre, notre contribution réside dans la prise en compte de la concentration spatiale de l'influence de la Politique de Cohésion (et d'autres facteurs explicatifs du développement régional) sur la croissance. Grâce à la méthode utilisée, nous pourrons ainsi identifier des groupes de régions (clubs spatiaux) où la polarisation économique ou au contraire la pauvreté persistent malgré les Fonds européens dépensés.

\section{Approche méthodologique}

\section{Données et variables}

Le niveau territorial considéré dans la présente étude est le niveau NUTS 2. Cette échelle régionale est appropriée aux fins de cette étude car elle nous permet d'obtenir des données pertinentes et comparables et de discuter et de traiter des recommandations politiques à un niveau institutionnel adéquat, compte-tenu que les Fonds européens sont distribués à cette échelle. Notre échantillon est constitué de 248 régions appartenant à 23 Etats-membres. Ainsi, la Croatie a été exclue car elle ne bénéficiait pas des Fonds européens pour la période étudiée, tout comme la Roumanie et la Bulgarie qui a reçu des Fonds de pré-adhésion sur la période 2000-2006 mais pas de Fonds européens issus de la Politique de Cohésion. Faute de disponibilité et de complétude des données, nous n'avons pas pu intégrer les chiffres relatifs aux programmes de pré-adhésion PHARE, ISPA et SAPARD. Enfin pour des raisons statistiques liées à des problématiques insulaires ou d'enclaves, Chypre, Malte, les Iles Canaries, les Açores, Madère et les Territoires d'Outre-Mer ont été également exclus.

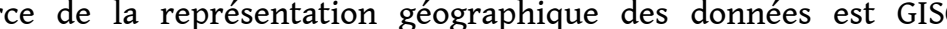
(Commission européenne) pour les limites administratives des régions européennes. 
Les données ont été récoltées via la DG Regio (pour celles concernant les Fonds européens) et Eurostat.

La croissance régionale de long terme (la variable dépendante considérée dans notre approche) est mesurée telles que dans le modèle de convergence de Barro et Sala-iMartin (1992) entre la période 2000 et 2016. Notons qu'une limite de notre travail réside dans son impossibilité de prise en compte de l'effet direct de la crise financière et immobilière de 2008 sur la croissance régionale de long terme que nous mesurons. Nous avons donc introduit un décalage temporel de quatre ans après la fin de celle-ci. Cet écart temporel est d'autant plus justifié qu'il permet de prendre en compte l'effet décalé de la Politique de Cohésion sur la croissance (puisque nous considérons les périodes de programmation 2000-2006 et 2007-2013). En effet, de nombreux auteurs ont déjà procédé à un décalage temporel pour éliminer les problèmes potentiels de causalité simultanée et reconnaissent que les investissements publics n'agissent pas instantanément sur la croissance (Mohl et Hagen, 2010 ; Sotiriou et Tsiapa, 2015 ; Mohl, 2016 ; Breidenbach et al., 2016 ; Dall'Erba, Fang, 2017).

Les variables explicatives de la croissance régionale de long terme sont choisies conformément aux travaux en science régionale sur l'évolution des disparités dans l'UE. Suivant les théories économiques dominantes de la croissance néoclassique d'une part (Solow, 1956; Swan, 1956) et l'approche de la croissance endogène d'autre part (Romer, 1986 ; Lucas, 1988), la croissance économique est le résultat d'une combinaison de trois facteurs que sont le capital physique, le capital humain (ou travail) et l'innovation (ou progrès technique). Plusieurs facteurs peuvent expliquer une répartition spatiale de la croissance économique qui est loin d'être homogène, comme l'accès aux marchés, le capital humain, le changement technologique, la compétitivité internationale, les économies d'échelle, l'efficacité institutionnelle et la localisation géographique. De fait, nous avons intégré dans notre approche exploratoire les variables indépendantes de la croissance décrites ci-après. Dans un premier temps,

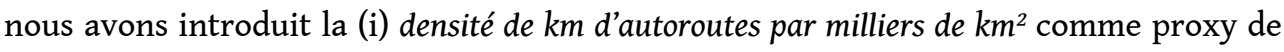
l'inégale répartition spatiale des activités économiques suivant les travaux issus de la géographie économique (Krugman 1991; Fujita, 1999). La littérature met en évidence que les régions les plus accessibles ont un marché potentiel plus élevé et sont davantage susceptibles de polariser les activités économiques et d'être plus attractives pour les investisseurs. Par conséquent, elles peuvent connaître des taux de croissance plus élevés (Krugman, 1991). Dans le même temps, d'autres auteurs ont montré dans le cas européen que la construction d'infrastructures est une condition nécessaire mais non suffisante à la croissance (Vickerman et al., 1999 ; Crescenzi, Rodríguez-Pose, $2008,2012)$. Les régions ayant de bonnes infrastructures de transport et qui sont bien connectées à des régions ayant des dotations similaires ont tendance à croître plus rapidement (cas des régions plutôt développées). Cependant, les investissements dans les infrastructures dans les régions périphériques les rendent plus vulnérables à la concurrence. En étudiant l'impact des Fonds européens au titre de l'objectif 1 sur la convergence, Rodríguez-Pose et Fratesi (2004) montrent que malgré la concentration des Fonds sur les infrastructures de transport, les rendements par rapport aux engagements consentis ne sont pas à la hauteur. La littérature sur l'effet de l'accessibilité d'une région sur sa croissance n'étant pas univoque, il semble donc intéressant de l'intégrer dans notre analyse. 
18 Dans un second temps, suivant les études académiques de nombreux chercheurs montrant les effets positifs et significatifs du capital humain sur la croissance économique (Lucas, 1998; Crescenzi, 2005 ; Ederveen et al., 2006; Faggian, McCann, 2009), nous avons intégré le niveau d'éducation de la population mesuré par la (ii) part de la population âgée de 25 à 64 ans étant diplômée de l'enseignement supérieur. Les modèles de croissance endogènes et schumpétériens mettent en évidence des problèmes de faibles niveaux de capital humain et de faible capacité à innover et assimiler les innovations en tant que facteurs limitant le potentiel de croissance. La géographie économique et l'économie de la croissance mettent l'accent sur l'innovation et les processus d'apprentissage - la création et l'assimilation des connaissances. Elles se rejoignent dans la mesure où le capital humain nécessaire pour innover opère au travers de réseaux de transmission et de formation du savoir. Ceci a des implications spatiales importantes car les coûts de transaction de la transmission des connaissances restent élevés, impliquant souvent des contacts en face à face, des canaux institutionnels définis et de longues périodes pour construire ces canaux. De plus, l'écart des capacités technologiques et d'innovation entre les régions - parfois attribué aux différences dans les niveaux de capital humain, parfois aux différences dans la capacité structurelle en R\&D - peut expliquer les écarts de développement et la concentration spatiale de la croissance régionale, et les éventuels effets de débordement associés. On retrouve ainsi de nombreux travaux relevant l'importance des investissements dans la R\&D pour favoriser le développement régional (Ederveen et al., 2006 ; Bachtler, Gorzelak, 2007 ; Camagni, Capello, 2013 ; Molle, 2015), justifiant l'intégration de la variable (iii) dépenses totales en $R \& D$ rapportées en PPA/hab.

Puis, comme l'indiquent les deux derniers Rapport sur la Cohésion (2014 et 2017) tout comme le rapport Barca (2009), il semblerait que le développement économique soit conditionné par les capacités institutionnelles des régions. Ceci est confirmé par des travaux récents sur la question (Arbia et al., 2010 ; Arbolino, Boffardi, 2017 ; Di Cataldo, Rodríguez-Pose, 2017 ; Di Vita, 2017 ; Rodríguez-Pose, Ketterer, 2018). Les institutions et une gouvernance de bonne qualité peuvent être définies comme étant caractérisées par «l'absence de corruption, une approche pratique de la politique de la concurrence et des marchés publics, un environnement juridique efficace et un système judiciaire indépendant et efficace. [...] une capacité institutionnelle et administrative forte, réduisant la charge administrative et améliorant la qualité de la législation " (Commission européenne, 2014). Nous avons donc repris (iv) l'indice de qualité institutionnelle (EQI) développé par Charron et Lapuente (2013) et Charron et al. (2015) qui évalue le contrôle de la corruption, le traitement impartial des citoyens et l'efficacité du gouvernement régional à partir d'une large enquête auprès des habitants de l'UE.

Enfin, puisque nous souhaitons explorer où la Politique de Cohésion semble mieux fonctionner qu'ailleurs, nous avons intégré (v) la somme des Fonds de la politique régionale dépensés (tous objectifs confondus) sur la période 2000-2013 ainsi que (vi) la somme des Fonds européens dépensés au titre de l'objectif 1. L'ajout de cette dernière variable est particulièrement intéressant car les Fonds au titre de l'obj. 1 visent justement le rattrapage économique des régions les plus en difficulté. De plus, comme Bourdin (2014) le souligne, l'intégration des PECO a bouleversé la répartition des Fonds structurels entre 2000-2006 et 2007-2013, puisque $53 \%$ des montants sont revenus aux pays entrants. Toutefois, en même temps, des aides transitoires ont été accordées aux 
régions qui se sont trouvées brusquement enrichies en moyenne (bien que toujours en retard) par l'intégration de régions en grande difficulté. Les effets de dépendance spatiale de ces Fonds ont largement été montrés dans la littérature. Par exemple, Dall'erba (2005) explique qu'une entreprise située dans une région ciblée par les Fonds structurels n'effectue pas nécessairement la construction d'une nouvelle infrastructure financée par ceux-ci. En conséquence, une partie de la valeur ajoutée d'un projet européen dans une région peut bénéficier également à d'autres régions. Tout comme cette nouvelle infrastructure (par exemple une autoroute) pourra davantage profiter à une région voisine (par exemple une région capitale) qu'à la région traitée pour des raisons de forces centripètes (comme expliqué par la nouvelle géographie économique).

\section{Analyse exploratoire des données spatiales bivariée}

21 Au travers de la méthodologie utilisée, l'objectif de notre papier n'est pas de réaliser une évaluation économétrique de l'efficacité de la politique régionale mais bien de mener une analyse exploratoire spatiale permettant d'identifier des régions ou groupes de régions qui « gagnent » ou qui " perdent» (Benko, Lipietz, 1992, 2000), en partant de l'hypothèse issue de la littérature selon laquelle il existe des effets spatiaux (dépendance spatiale et hétérogénéité spatiale) des relations entre la croissance régionale et ses facteurs explicatifs (dont les Fonds européens sur lesquels nous nous focalisons).

Une technique de l'analyse de co-localisation a été développée par Anselin et al. (2002) qui a conçu un indicateur local d'association spatiale bivarié (BiLISA) pour étudier le modèle de corrélation spatiale entre deux variables géoréférencées (ie. nombre de points de vente d'alcool par rapport au nombre d'incidents criminels dans les zones concernées). Les BiLISA permettent d'évaluer la corrélation entre une variable dans une région et l'autre variable dans les régions voisines. L'analyse bivariée permet d'isoler la relation entre la variable dépendante et les variables explicatives une à une. Il est également possible d'étudier l'autocorrélation spatio-temporelle, autrement dit, la corrélation d'une variable en référence à la localisation spatiale d'une seule variable dans un intervalle de temps. On analyse alors la corrélation d'une variable avec ellemême dans l'espace et le temps (Anselin et al., 2002). L'idée ici est d'examiner de manière paramétrique (au-delà de l'inspection visuelle), comment les schémas spatiaux sont en corrélation entre plusieurs variables (Wartenberg, 1985). Il s'agit alors de tirer des conclusions sur la dynamique spatiale qui relie un ensemble de données localisées. En termes plus techniques, l'examen de la similitude des processus spatiaux entre variables peut être considéré comme un moyen de tester la robustesse ou la persistance dans le temps d'un modèle/schéma spatial donné, par exemple en comparant les modèles d'association spatiale locale entre la croissance économique et le niveau de développement initial (robustesse) ou entre les niveaux de développement régionaux à deux périodes raisonnablement distancées dans le temps (persistance). Ainsi, la méthode utilisée permet de capter les covariations spatiales des facteurs explicatifs à l'origine des phénomènes de convergence ou de divergence dans l'UE. Il est donc possible d'identifier si pour chaque région, le taux de croissance est significativement corrélé dans l'espace avec une autre variable. En revanche, étant donné qu'elle analyse des co-variations spatiales de variables deux à deux, la méthode utilisée ne permet pas d'analyse multivariée de ces co-variations spatiales et donc de déterminer de manière 
paramétrique les régions cumulant toutes les difficultés (faible niveau de développement, faible croissance, faible niveau d'éducation, faible niveau de qualité institutionnel, etc.) malgré la somme de Fonds européens dépensés.

Afin d'identifier et d'évaluer l'ampleur des relations spatiales, nous avons utilisé l'I de Moran pour mesurer l'autocorrélation spatiale et identifier les clusters spatiaux dans les données. Pour notre étude, quatre types d'associations spatiales peuvent être dérivées de cette statistique, avec des types high-high ( $\mathrm{HH}$ - concentration spatiale de valeurs fortes de la croissance et de valeurs fortes de la variable indépendante des régions voisines) et low-low ( $\mathrm{LL}$ - concentration spatiale de valeurs faibles de la croissance et de valeurs faibles de la variable indépendante des régions voisines) pour le regroupement spatial de valeurs similaires et les types high-low (HL - concentration spatiale de valeurs fortes de la croissance et de valeurs faibles de la variable indépendante des régions voisines) et low-high ( $\mathrm{LH}$ - concentration spatiale de valeurs faibles de la croissance et de valeurs fortes de la variable indépendante des régions voisines) pour le regroupement spatial de valeurs dissemblables.

La mesure d'autocorrélation spatiale locale bivariée utilisant la statistique I de Moran, dérivée de la formule d'Anselin (1995), s'écrit :

$I=z_{x i} \sum_{j=1, j \neq i}^{N} w_{i j} z_{y j}$

où $x_{i}$ représente la croissance régionale et $y_{j}$ la variable indépendante pour la région $i$ et la région voisine $j$; et $z_{x}$ et $z_{y}$ sont les z-scores standardisés des variables $x_{i}$ et $y_{j}$. Le choix de la matrice de poids spatial $w_{i j}$ modélisant la proximité spatiale et l'interdépendance entre les unités régionales est un sujet difficile et controversé dans les analyses spatiales. Il existe un large éventail d'applications pour déterminer $\mathrm{W}$ selon le but des études (Anselin et al., 2000, Shi et al., 2006) et GeoDa fournit désormais le seuil au-delà duquel la corrélation spatiale se finit, autrement dit, le seuil de distance au-delà duquel les valeurs de paires de voisins ne sont plus corrélées. Dans cette étude, une matrice a été calculée selon le critère de distance euclidienne-seuil de $323 \mathrm{~km}$.

Une approche de permutations (ici 999) a été utilisée pour évaluer la significativité statistique des résultats de l'I de Moran et les BiLISA. La randomisation suppose que l'emplacement des valeurs et leur disposition spatiale sont sans importance. Basé sur la randomisation, différents écarts-types théoriques pour I de Moran sont obtenus, chacun donnant une p-valeur différente comme une pseudo-significativité. La valeur de seuil de $p=0,05$ définit la significativité. Les $p$-valeurs suivent une distribution asymptotiquement standard-normale qui permet l'évaluation de leur niveau de signification en les comparant à une distribution de référence (Anselin 1995), et donc de définir les seuils au-delà desquels les relations $\mathrm{HH}, \mathrm{LL}, \mathrm{LH}, \mathrm{HL}$ ne sont plus significatives. Les BiLISA ont été calculés à l'aide du logiciel GeoDa version 1.12 (Anselin et al., 2006). 


\section{Analyse spatiale exploratoire des facteurs explicatifs de la croissance régionale et de leur dépendance}

L'objectif est de mener une analyse exploratoire permettant de mettre en évidence des regroupements spatiaux au sein desquels il y aurait une concentration de régions qui cumuleraient des difficultés ou des avantages quant à leur développement. Nous avons mené une approche en deux temps. Tout d'abord, nous avons commencé par analyser les co-localisations de concentrations spatiales entre la croissance régionale et le PIB/ hab. à l'année initiale de notre étude (en 2000); ceci a permis de dégager les concentrations spatiales de régions qui convergent et qui divergent (entre 2000 et 2016). Ensuite, nous avons analysé les co-localisations de concentrations spatiales entre la croissance régionale et les variables explicatives. Il a été ainsi possible de mettre en regard les concentrations spatiales de régions qui convergent/divergent avec les concentrations spatiales des facteurs explicatifs à l'origine de l'évolution des disparités.

\section{Les trajectoires de la croissance régionale : divergence et effets de rattrapage}

En cartographiant les résultats, il est possible d'identifier les concentrations spatiales de régions qui ont connu un processus de rattrapage sur la période $(\mathrm{HL}$ - régions qui ont connu une forte croissance et dont les voisines avaient un niveau de développement économique initial bas) et les régions qui ont divergé ( $\mathrm{LL}$ - régions qui ont enregistré un taux de croissance faible et dont les voisines étaient peu développées en 2000). La figure 1 montre très clairement l'existence de clubs spatiaux de convergence ou de divergence avec les régions des PECO qui font partie des régions qui gagnent tandis que les régions de l'Europe méditerranéenne (sud de l'Italie et Grèce) sont les régions qui perdent. Ce premier constat nous permet de montrer que des régions en retard (Europe méditerranéenne et Europe centrale et orientale) peuvent avoir des trajectoires régionales de croissance différentes confirmant les travaux récents de Iammarino et al. (2017). Par la suite, l'étude des co-localisations entre la croissance régionale et les variables explicatives identifiées nous permettra d'apporter des éclairages sur l'explication de ces schémas spatiaux. La figure 1 met en lumière la «banane bleue » de Roger Brunet (2002) via les regroupements spatiaux (LH - régions avec une croissance économique faible mais un niveau de développement économique en 2000 élevé). Cette dorsale européenne s'étend de Londres à Milan et est considérée comme un espace économique majeur, densément peuplé et fortement urbanisé. Dans cette dorsale, on retrouve des régions métropolitaines particulièrement dynamiques (HH - régions avec une croissance économique forte et un niveau de développement économique en 2000 élevé) qui correspondent sans surprise à des capitales et à l'Allemagne de l'Ouest. 
Figure 1. Les trajectoires régionales de la croissance régionale et du développement économique.

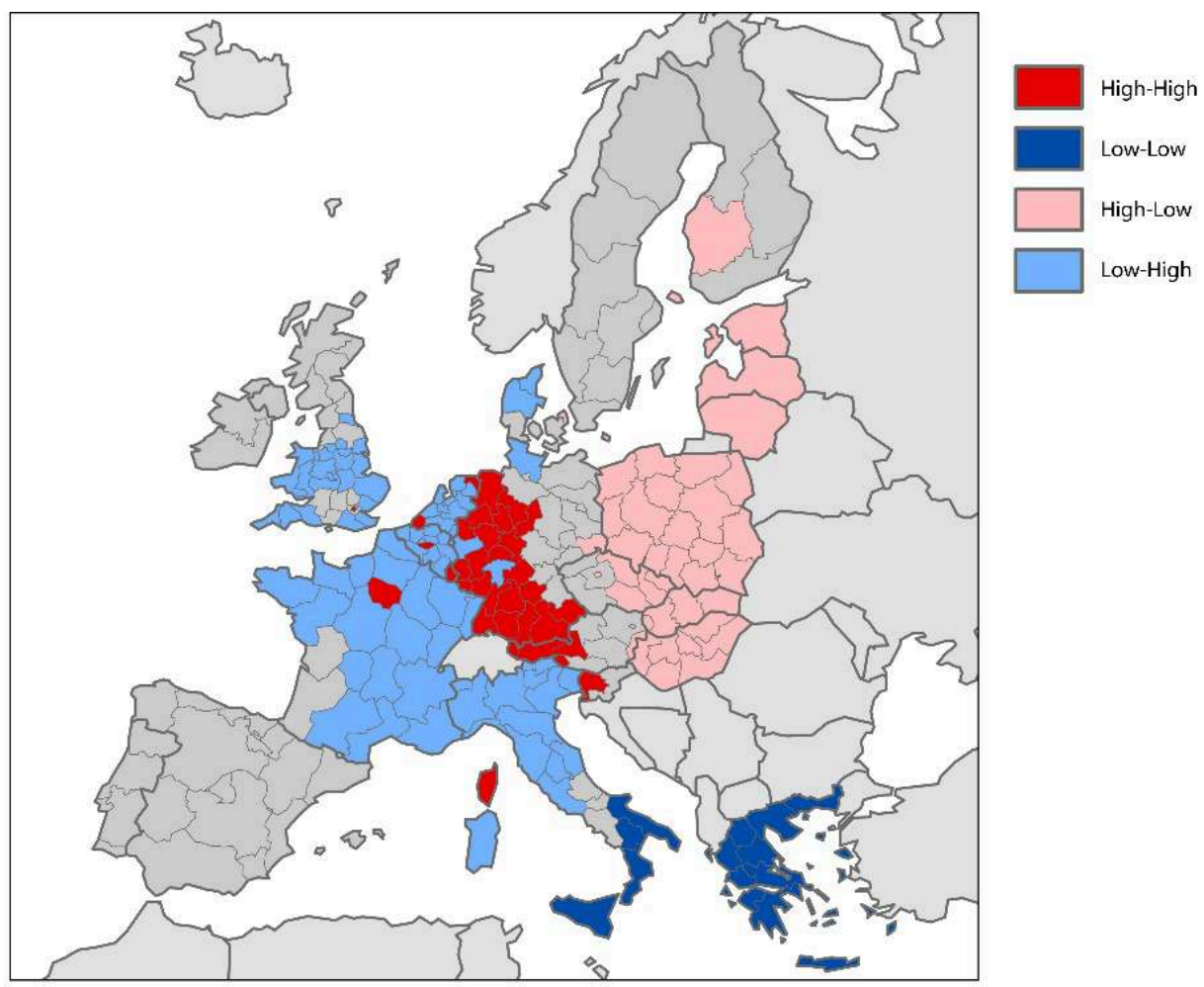

\section{Concentrations spatiales de la croissance régionale et de ses facteurs explicatifs : les trappes de développement dans l'Union européenne}

Comme Borderon et Oliveau (2016) l'expliquent, la cartographie des BiLISA permet d'identifier des pièges spatiaux, or, pour notre cas d'étude, cartographier les colocalisations des taux de croissance enregistrés avec d'autres variables nous permet de mettre en évidence (i) des trappes de développement regroupant des régions voisines qui cumulent des difficultés (il peut s'agir par exemple d'une concentration spatiale de régions où les Fonds européens ne semblent pas avoir les effets escomptés car ces régions ont reçu et dépensé beaucoup d'argent mais leur croissance et celle de leurs voisines reste faible) ou au contraire (ii) des zones dans l'UE qui ont tiré profit des Fonds européens en améliorant de manière significative leur niveau de développement en investissant dans les facteurs les plus susceptibles de créer de la croissance. Notre approche permet donc d'identifier les concentrations spatiales de régions cumulant des difficultés et qui nécessiteraient, dès lors, une plus grande attention des décideurs politiques européens. Notons que, du fait de son caractère exploratoire, cette méthode ne nous permet cependant pas d'identifier des relations causales qui existeraient entre les différents explicatifs de la croissance régionale dans l'UE. 
Figure 2. Concentrations spatiales de la croissance régionale et de ses facteurs explicatifs.

Carte a. : Croissance régionale et Total des Fonds de la Politique de Cohésion

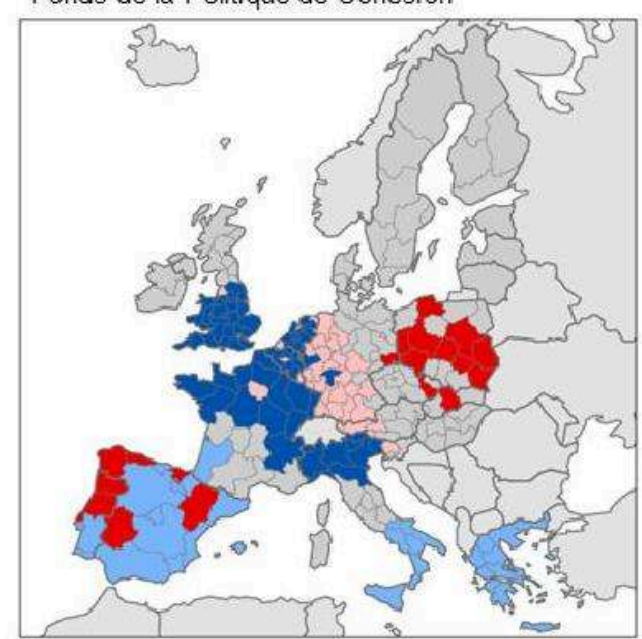

Carte c. : Croissance régionale et qualité institutionnelle

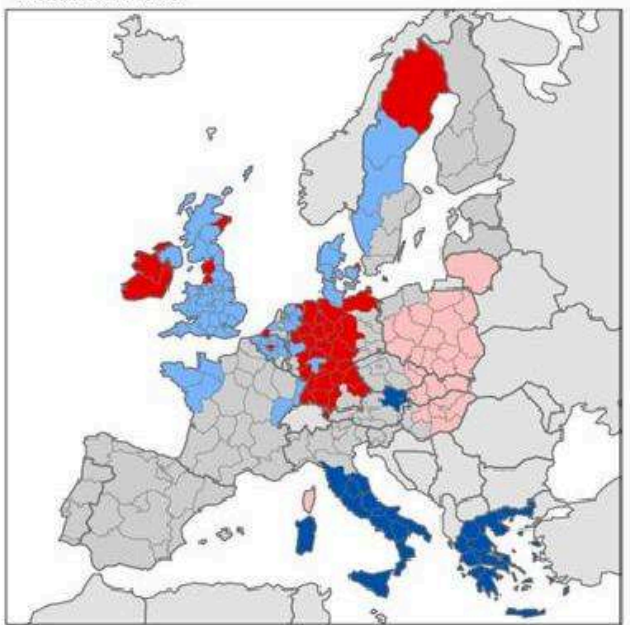

Carte e : Croissance régionale et niveau d'éducation

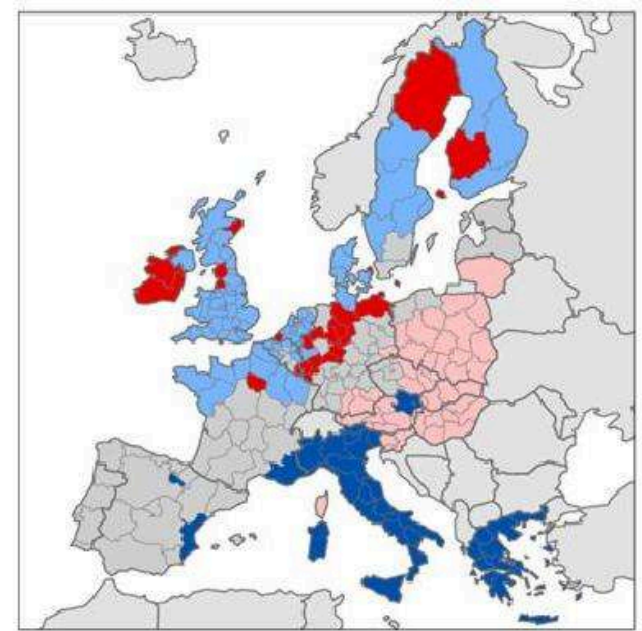

Carte b. : Croissance régionale et Fands européens Obj. 1

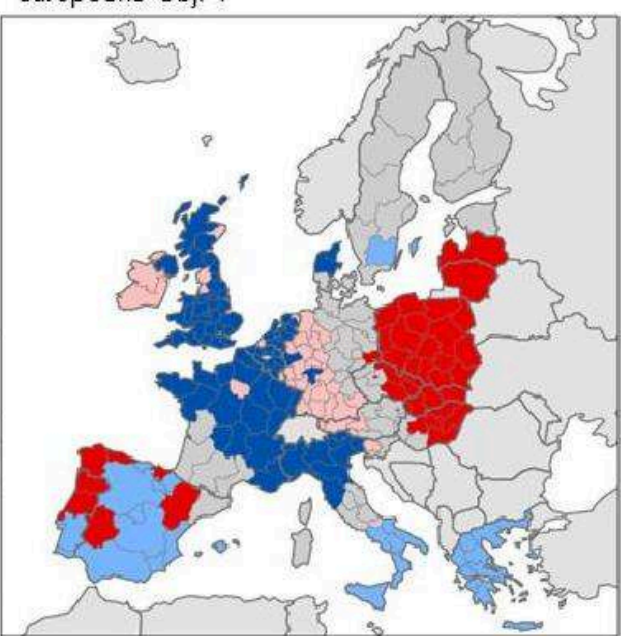

Carte d. : Croissance régionale et accessibilité

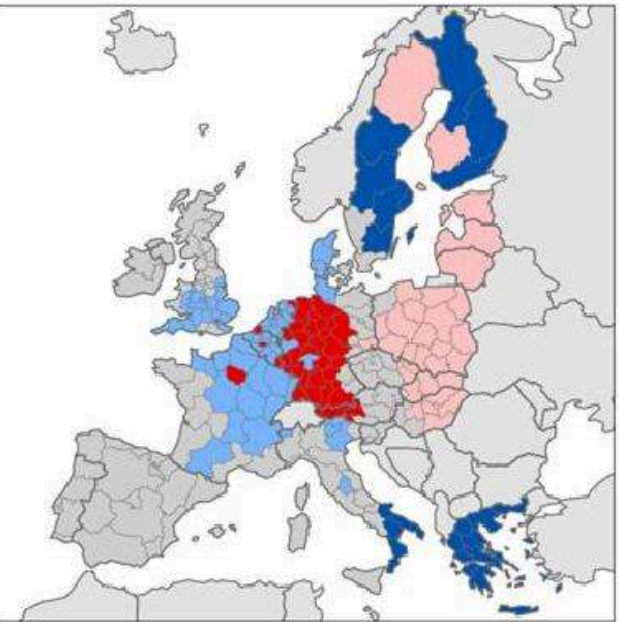

Cartef. : Croissance régionale et $R \& D$

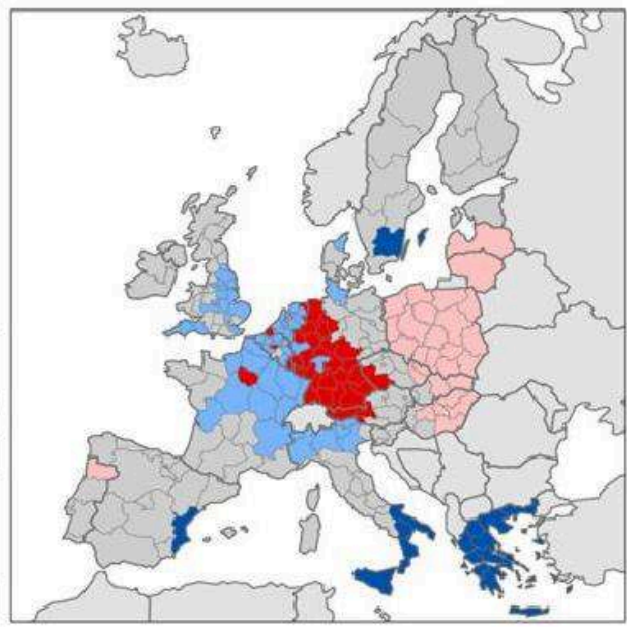


La cartographie des BiLISA (figure 2) permet d'identifier différents schémas spatiaux. Tout d'abord, sur la carte $b$, les zones en rouge peuvent-être considérées comme une concentration spatiale de régions qui gagnent où l'on peut supposer que les Fonds européens ont été utilisés efficacement. Les régions qui composent ces zones ont connu des taux de croissance élevés en lien avec les montants importants reçus et dépensés par elles et leurs voisines sur la période considérée (carte a). Dans l'ensemble, pour ces régions, les investissements ont porté leurs fruits en termes de convergence (Cappelen et al., 2003 ; Becker et al., 2010 ; Pellegrini et al., 2013 ; Bourdin, 2018). Dans ces grappes de régions on retrouve une large partie des régions occidentales de la péninsule ibérique, en particulier le Portugal, confirmant les travaux antérieurs (Becker et al., 2010 ; Bachtler, Turok, 2013) mais aussi les régions centre-orientales, en particulier les voïvoidies polonais. Pour ces régions, l'analyse des cartes c à f exprime largement un effet de rattrapage puisque ces régions avaient, à la période initiale, des niveaux bas en terme de qualité institutionnelle, d'accessibilité, de formation du capital humain et d'investissements dans la R\&D (cartes c, d, e, f). Ajoutons qu'outre le fait que ces régions ont été les premières bénéficiaires des Fonds européens parmi toutes les régions européennes, on peut faire l'hypothèse que leur croissance économique peut aussi s'expliquer par des effets de débordement géographique liés à leur proximité avec les Länder allemands (Bański, 2010 ; Bourdin , 2015).

Ensuite, à l'inverse, en bleu ciel sur la carte b, on retrouve une concentration spatiale de régions qui perdent. Malgré le fait qu'elles aient reçu des enveloppes importantes issues de la Politique de Cohésion (carte a - concentration spatiale de valeurs faibles de la croissance et de valeurs fortes des Fonds européens reçus et dépensés des régions voisines), elles n'ont visiblement pas connu les taux de croissance espérés compte-tenu des sommes d'argent mobilisées. Dès lors, on peut s'interroger sur les raisons de la baisse apparente du rendement de l'investissement. En observant la carte c, on peut trouver des premiers éléments de réponse, qui constituent à ce stade que de simples hypothèses car nous ne les testons pas économétriquement dans cet article. Un certain nombre d'études récentes (Rodríguez-Pose, Garcilazo, 2015 ; Ketterer, Rodríguez-Pose, 2016) et le dernier rapport sur la cohésion économique, sociale et territoriale de la Commission européenne (2017) indiquent que la bonne gouvernance affecte la croissance économique et la qualité de la vie. Il est relevé l'importance de ce facteur dans la performance économique des régions et le fait qu'une gouvernance de mauvaise qualité dans les zones défavorisées de l'UE représentait un obstacle important au développement. En effet, il a été constaté non seulement que cela affecte négativement la croissance économique, mais aussi les retours sur investissement de la Politique de Cohésion et la compétitivité régionale (Rodríguez-Pose, Di Cataldo, 2015). Dans le même temps, il a été mis en évidence qu'un gouvernement corrompu ou inefficace remettait en cause le potentiel régional d'innovation et d'entrepreneuriat (Nistotskaya et al. 2015 ; Annoni, Dijkstra, 2017). La carte $\mathrm{f}$ semble confirmer cette hypothèse, où l'on observe une concentration spatiale forte de régions ayant enregistré des taux de croissance bas et dont les voisines ont un niveau d'éducation faible (en bleu sur la carte f), excepté pour Athènes (en rose sur la carte f). L'indice de qualité de la gouvernance développé par Charron et al. (2012) et mis à jour dans le dernier rapport sur la Cohésion (2017) montre très clairement que les régions présentant une qualité de gouvernance insuffisante sont localisées en Roumanie et Bulgarie, en Grèce et dans le sud de l'Italie. Or, ces dernières font partie des régions identifiées en bleu ciel dans les cartes a et $b$. On peut donc s'interroger sur la façon dont sont dépensés les Fonds européens dans ces 
régions, compte-tenu de leur faible élasticité. A cet effet, l'analyse de la carte $\mathrm{d}$ montre bien que les mêmes causes ne produisent pas les mêmes effets. Alors que les régions sud-méditerranéennes et centre-orientales étaient, en 2000, mal desservies, les premières n'ont pas su sortir de leurs pièges spatiaux tandis que les secondes ont connu une croissance de long terme élevée entre 2000 et 2016. L'amélioration de l'accessibilité pour les régions de l'Est (grâce notamment aux Fonds européens dépensés pour la politique des transports) a été un levier de développement (figure 2d). Le désenclavement a facilité l'attraction d'investissements directs à l'étranger dans ces territoires (Kelly et al., 2015). Les travaux récents de Rosik et al. (2015) confirment, pour le cas polonais, la place déterminante des Fonds européens pour développer les infrastructures de transport. Selon les auteurs, la réduction des disparités en matière d'accessibilité aux niveaux européen, national et régional peut déboucher non seulement sur un meilleur accès à la «banane bleue » dans le noyau européen mais également sur un meilleur accès aux centres économiques nationaux et régionaux. Suivant la théorie de la géographie économique et liés à la question des transports, les efforts en $R \& D$ génèrent également des retombées de connaissances dans les régions voisines localisées dans les limites fonctionnelles de l'économie régionale (Sonn, Storper, 2008; Bronzini, Piselli, 2009), faisant de la connectivité des transports un moyen pertinent de diffusion des connaissances. Comme les connaissances spécialisées sont généralement détenues par un nombre limité de personnes, il est difficile de les transmettre sans interactions et collaborations directes (Storper, Venables, 2004) et l'infrastructure de transport facilite la diffusion des connaissances entre les personnes et les entreprises dans une zone définie. L'investissement dans la R\&D dans son ensemble et les investissements dans la R\&D et dans l'enseignement supérieur dans les régions périphériques de l'UE sont positivement associés à l'innovation (Bilbao-Osorio, Rodríguez-Pose, 2004). Cependant, la force de cette relation repose sur les capacités spécifiques de la région à transformer l'investissement en R\&D en innovation (Camagni, Capello, 2013). De ce point de vue, les régions centre-orientales semblent bien s'en sortir alors que les régions italiennes septentrionales et les régions grecques n'ont pas connu une augmentation significative de leur croissance liée à une politique de développement du capital humain (figures $2 \mathrm{e}$ et $2 \mathrm{f}$ ).

31 Les régions localisées dans les zones en bleu foncé sur la carte b sont quant à elles caractérisées par des taux de croissance relativement faibles (mais des niveaux de développement élevés en 2000) et ont reçu peu de Fonds européens (carte a). On retrouve ici les régions de la dorsale européenne telle qu'identifiée dans la figure 1. Elles sont dans l'ensemble très accessibles, ont une population bien éduquée et investissent dans l'innovation. Il s'agit bien souvent de régions dont le niveau de développement est situé proche de la moyenne de l'UE ou au-delà ; et donc exemptes de la plus grosse part de l'enveloppe de la Politique de Cohésion au titre de l'objectif 1 (celle-ci étant attribuée pour les régions ayant un PIB/hab. inf. à $75 \%$ de la moyenne de l'UE).

32 Enfin, en rose clair sur la carte b, on identifie des clusters de régions en tête qui ont connu des taux de croissance élevés mais n'ont pas bénéficié de larges sommes de la politique régionale du fait de leur niveau de richesse élevé. On retrouve en toute logique les régions capitales (centre-Londres, Bruxelles-Capitale, Zeeland et Ile-deFrance) qui bénéficient des économies d'agglomération (Geppert, Stephan, 2008), c'est également le cas des Länder de l'ouest. 


\section{Conclusion et discussion}

liées à la croissance régionale et en particulier aux dépenses de Fonds européens en utilisant une méthode d'analyse spatiale qui reste encore peu utilisée à ce jour. Notre but était d'interroger les concentrations spatiales des relations qui existaient entre la croissance régionale et d'autres variables en prenant en compte l'absence d'indépendance entre des observations géographiques. En ce sens, les BiLISA permettent à la fois (i) d'identifier des trappes de développement ou des clubs spatiaux de régions «qui gagnent » et (ii) de mettre en lumière des trajectoires régionales de convergence/divergence que l'on peut mettre en regard avec différents facteurs explicatifs. Les résultats montrent que, à l'hétérogénéité spatiale du développement régional - qui a été largement montrée dans la littérature - vient s'ajouter une influence spatialement différenciée des Fonds européens, posant alors la question morale/politique d'une solidarité éventuelle entre les régions et appelant une nécessaire refonte de la Politique de Cohésion. Sans permettre de déterminer des relations causales entre les variables identifiées et la croissance régionale, notre méthode permet néanmoins d'identifier les concentrations spatiales de régions qui connaissent une forte (relativement faible) croissance et dont les voisines ont des niveaux forts (et/ou relativement faibles) d'une autre variable. L'étude a permis de mettre en évidence des régions cumulant les difficultés ou au contraire les avantages, avec la limite que la méthode ne permet pas une analyse multivariée de la colocalisation de concentrations spatiales de plus de deux variables.

Ces dernières années, les recherches sur l'évaluation ex-post de l'impact de la Politique de Cohésion se sont multipliées. Elles mettent en lumière les circonstances à l'origine de l'impact spatialement différencié des Fonds structurels (Crescenzi, Giua, 2017). Les résultats des travaux sur la question montrent que l'efficacité de cette politique est conditionnée par (i) la concentration des Fonds sur certaines thématiques, par (ii) l'environnement régional, (iii) par la bonne gouvernance et par (iv) les spécificités régionales locales/le capital territorial de la région. Du fait de son aspect exploratoire, loin de pouvoir démontrer à partir de l'utilisation des BiLISA des causalités entre les Fonds européens et la croissance économique observée dans certaines régions, notre étude confirme néanmoins l'état de l'art sur la question en montrant les influences différenciées de la Politique de Cohésion - tout comme celles de l'accessibilité, la qualité institutionnelle, l'éducation, etc. - sur la croissance des régions. L'intérêt de cette approche est qu'elle permet (i) d'identifier des trajectoires régionales de développement économique, (ii) de comprendre les facteurs sous-jacents à celles-ci et (iii) de circonscrire spatialement les clubs de régions qui gagnent (régions centreorientales pour une grande partie, et dans une moindre mesure un certain nombre de régions de la péninsule espagnole) ou au contraire qui perdent (les régions du sud de l'Italie et les régions grecques à l'exception de la région Attique).

De facto, ces résultats abondent dans le sens des approches modernes du développement territorial qui prennent en compte le rôle clé de la géographie dans les politiques ciblant la croissance économique. A partir de notre analyse, il semblerait alors raisonnable de penser, par exemple, qu'être une région moins développée parmi les zones les moins développées n'a pas la même incidence que d'être moins développée 
parmi des voisins prospères. Dès lors, nos résultats confirment l'approche soutenue par l'OCDE (2009) qui consiste à développer les potentiels de croissance qui existent dans chaque région et, à mettre en œuvre des politiques de développement territorial qui visent à aider les régions en retard en investissant dans des secteurs clés susceptibles de créer de la croissance, mais aussi en mettant tout en œuvre pour améliorer leur bonne gouvernance. Ces nouvelles politiques appelées "place sensitive policies» et "place-based policies» permettront de mieux cibler les régions et le type d'aides appropriées pour favoriser le développement territorial.

Dans ce cadre, tout comme nos résultats le montrent, l'approche descendante du « onesize-fits-all » de la Politique de Cohésion a trouvé ses limites. C'est pour cette raison que la Politique de Cohésion 2014-2020 inclut désormais une dimension territorialisée qui implique de i) se concentrer sur les avantages locaux endogènes et la connaissance, ii) concevoir et adapter les interventions aux contextes spécifiques et à leurs liens spatiaux, iii) stimuler les choix et orientations des acteurs locaux (Barca, 2009). Il nécessite également de donner un poids plus important aux niveaux infranationaux (Leonardi, 2005) via la spécialisation intelligente, considérée comme un facteur clé pour lutter contre les disparités économiques dans les régions européennes (Commission européenne, 2010).

Dans un contexte de Brexit et de négociations actuelles tendues sur le futur budget de la Politique de Cohésion post-2020, notre étude exploratoire appelle certainement à un réexamen des mécanismes de prise de décision existants dans ce domaine et à une évaluation plus rigoureuse des projets financés pour des raisons d'opportunité/de coût. Ainsi, en période de pénurie des ressources publiques, il faudra peut-être repenser comment améliorer l'efficacité (le rapport entre les résultats obtenus et les objectifs fixés par les décideurs européens et régionaux) et l'efficience (l'optimisation des outils/ moyens mis en œuvre pour obtenir un résultat) des politiques publiques de développement territorial européen (Bourdin, Ragazzi, 2018). Ceci passe probablement par un accompagnement des régions vers une meilleure gouvernance d'une part, et par la mise en œuvre de politiques de développement plus intégrées et plus inclusives fondées sur le capital humain et l'innovation d'autre part. Les travaux académiques montrent qu'elles seules entraînent des rendements plus importants des Fonds publics, mais aussi une plus grande durabilité de l'effort de développement.

\section{BIBLIOGRAPHIE}

ANNONI P., DIJKSTRA L. (2017), “Measuring and monitoring regional competitiveness in the European Union”, in HUGGINS R., THOMPSON P. (eds.), Handbook of Regions and Competitiveness Contemporary Theories and Perspectives on Economic Development, Edward Elgar Publishing. ANSELIN L. (2000), “Spatial Econometrics”, in BALTAGI B. (ed.), Companion to Econometrics, Basil Blackwell. 
ANSELIN L. (1995), "Local indicators of spatial association-LISA", Geographical analysis, 27, 2, pp. 93-115.

ANSELIN L., COHEN J., COOK D., GORR W. \& TITA G. (2000), “Spatial analyses of crime”, Criminal justice, 4, 2, pp. 213-262.

ANSELIN L., SYABRI I. \& KHO Y. (2006), “GeoDa : an introduction to spatial data analysis”, Geographical analysis, 38, 1, pp. 5-22.

ANSELIN L., SYABRI I. \& SMIRNOV O. (2002), "Visualizing multivariate spatial correlation with dynamically linked windows", Urbana, 51, 61801.

ARBIA G., BATTISTI M. \& DI VAIO G. (2010), “Institutions and geography : Empirical test of spatial growth models for European regions”, Economic Modelling, 27, 1, pp. 12-21.

ARBOLINO R., BOFFARDI R. (2017), “The Impact of Institutional Quality and Efficient Cohesion Investments on Economic Growth Evidence from Italian Regions", Sustainability, 9, 8, p. 1432.

ARISTEI D., PERUGINI C. (2012), "Inequality and reforms in transition countries”, Economic Systems, 36, 1, pp. 2-10.

BACHTLER J., GORZELAK G. (2007), "Reforming EU Cohesion Policy : A reappraisal of the performance of the Structural Funds", Policy studies, 28, 4, pp. 309-326.

BACHTLER J., TUROK I. (eds.), (2013), The coherence of EU regional policy: Contrasting perspectives on the Structural Funds, Routledge.

BACHTLER J., WREN C. (2006), “Evaluation of European Union Cohesion policy : Research questions and policy challenges”, Regional studies, 40, 2, pp. 143-153.

BAŃSKI J. (2010), “Dilemmas for regional development in the concepts seeking to develop Poland's spatial structure", Regional Studies 44, 5, pp. 535- 554

BARCA F., (2009), Agenda for a Reformed Cohesion Policy ; a place-based approach to meeting European Union challenges and expectations, European Communities.

BARRO R. J., SALA-I-MARTIN X. (1992), “Convergence”, Journal of political Economy, 100, 2, pp. 223-251.

BASLÉ M. (2006), “Strengths and weaknesses of European Union policy evaluation methods : expost evaluation of Objective 2, 1994-99”, Regional Studies, 40, 2, pp. 225-235.

BECKER S. O., EGGER P.H. \& VON EHRLICH M. (2010), “Going NUTS : The effect of EU Structural Funds on regional performance", Journal of Public Economics, 94, 9-10, pp. 78-590.

BECKER S. O., EGGER P. H. \& VON EHRLICH M. (2012), "Too much of a good thing ? On the growth effects of the EU's regional policy", European Economic Review, 56, 4, pp. 648-668.

BENKO G., LIPIETZ A. (1992), Les régions qui gagnent, Paris, Puf.

BENKO G., LIPIETZ A. (2000), La richesse des régions : la nouvelle géographie socio-économique, Presses universitaires de France.

BILBAO-OSORIO B., RODRÍGUEZ-POSE A. (2004), "From R\&D to innovation and economic growth in the EU", Growth and Change, 35, 4, pp. 434-455.

BORDERON M., OLIVEAU S. (2016), “Vulnérabilités sociales et changement d'échelle. L'espace compte!", Espace populations sociétés, 3. 
BOUAYAD-AGHA S., TURPIN N. \& VÉDRINE L. (2013), “Fostering the development of European regions : A spatial dynamic panel data analysis of the impact of cohesion policy", Regional Studies, 47,9 , pp. 1573-1593.

BOURDIN S. (2010), “Repenser le futur de la politique de cohésion européenne pour les pays d'Europe centrale et orientale : la prise en compte de l'espace géographique comme piste de recherche", Bulletin de la Société géographique de Liège, 55, pp. 43-59.

BOURDIN S. (2014), Les défis de la future politique régionale européenne 2014-2020 : on prend les mêmes et on recommence?, Geoconfluences.

BOURDIN S. (2015), "National and regional trajectories of convergence and economic integration in Central and Eastern Europe”, Canadian Journal of Regional Science, 38, 1/3, pp. 55-63.

BOURDIN S. (2018), “Analyse spatiale de l'efficacité des Fonds structurels européens sur la croissance régionale”, Revue d'Économie Régionale \& Urbaine, 2, pp. 243-270.

BOURDIN S., RAGAZZI E. (2018), "La science régionale et la performance des politiques publiques : retour sur les méthodes d'évaluation”, Revue d'Économie Régionale \& Urbaine, 2, pp. 225-242.

BREIDENBACH P., MITZE T. \& SCHMIDT C.M. (2016), EU structural funds and regional income convergence-A sobering experience, Working paper.

BRONZINI R., PISELLI P. (2009), “Determinants of long-run regional productivity : the role of R\&D, human capital and public infrastructure", Regional Science and Urban Economics, 39, 2, pp. 187-199.

BRUNET R. (2002), “Lignes de force de l'espace Européen”, Mappemonde, 66, 2, pp. 14-19.

CAMAGNI R. (2002), “Compétitivité territoriale, milieux locaux et apprentissage collectif : une contre-réflexion critique”, Revue d'Economie Régionale \& Urbaine, 4, pp. 553-578.

CAMAGNI R., CAPELLO R. (2013), "Regional innovation patterns and the EU regional policy reform : Toward smart innovation policies" Growth and change, 44, 2, pp. 355-389.

CAPPELEN A., CASTELLACCI F., FAGERBERG J. \& VERSPAGEN B. (2003), “The impact of EU regional support on growth and convergence in the European Union", JCMS : Journal of Common Market Studies, 41, 4, pp. 621-644.

CARRINGTON A. (2003), “A divided Europe? Regional convergence and neighbourhood spillover effects”, Kyklos, 56, 3, pp. 381-393.

CHARRON N., LAPUENTE V. (2013), "Why do some regions in Europe have a higher quality of government?", The Journal of Politics, 75, 3, pp. 567-582.

CHARRON N., DIJKSTRA L. \& LAPUENTE V. (2015), "Mapping the regional divide in Europe : A measure for assessing quality of government in 206 European regions", Social Indicators Research, 122, 2, pp. 315-346.

CLIFF A., ORD K. (1972), “Testing for spatial autocorrelation among regression residuals”, Geographical analysis, 4, 3, pp. 267-284.

COMMISSION EUROPÉENNE (2010), Ex post evaluation of Cohesion Policy Programmes 2000-06 cofinanced by the ERDF (Objective $1 \& 2$ ) : Synthesis report, Office des Publications, Luxembourg COMMISSION EUROPÉENNE (2014), $6^{\text {ème }}$ rapport sur la cohésion économique, sociale et territoriale, Office des Publications européennes

COMMISSION EUROPÉENNE (2017), 7 7̀me rapport sur la cohésion économique, sociale et territoriale, Office des Publications européennes 
CRESCENZI R. (2005), "Innovation and regional growth in the enlarged Europe : the role of local innovative capabilities, peripherality, and education", Growth and Change, 36, 4, pp. 471-507.

CRESCENZI R., GIUA M. (2016), “The EU Cohesion Policy in context : Does a bottom-up approach work in all regions ?", Environment and Planning A, 48, 11, pp. 2340-2357.

CRESCENZI R., RODRÍGUEZ-POSE A. (2008), "Infrastructure endowment and investment as determinants of regional growth in the European Union”, Eib Papers, 13, 2, pp. 62-101.

CRESCENZI R., RODRÍGUEZ-POSE A. (2012), "Infrastructure and regional growth in the European Union”, Papers in regional science, 91, 3, pp. 487-513.

DALL'ERBA S. (2005), “Distribution of regional income and regional funds in Europe 1989-1999: an exploratory spatial data analysis", The Annals of Regional Science, 39, 1, pp. 121-148.

DALL'ERBA S., FANG F. (2017), "Meta-analysis of the impact of European Union Structural Funds on regional growth”, Regional Studies, 51, 6, pp. 822-832.

DALL'ERBA S., LE GALLO J. (2007), “The impact of EU regional support on growth and employment", Czech Journal of Economics and Finance, 57, 7, pp. 325-340.

DALL'ERBA S., LE GALLO J. (2008), "Regional convergence and the impact of European structural funds over 1989-1999 : A spatial econometric analysis”, Papers in Regional Science, 87, 2, pp. 219-244.

DALL'ERBA S., GUILLAIN R. \& LE GALLO J. (2009), “Impact of structural funds on regional growth : how to reconsider a 9 year-old black box”, Région et Développement, 30, pp. 77-99.

DI CATALDO M., RODRÍGUEZ-POSE A. (2017), "What drives employment growth and social inclusion in the regions of the European Union?", Regional Studies, 51, 12, pp. 1840-1859.

DI VITA G. (2017), "Institutional quality and the growth rates of the Italian regions : The costs of regulatory complexity", Papers in Regional Science.

EDERVEEN S., GROOT H. L. \& NAHUIS R. (2006), "Fertile soil for structural funds ? A panel data analysis of the conditional effectiveness of European cohesion policy", Kyklos, 59, 1, pp. 17-42.

ERTUR C., KOCH W. (2005), “Une analyse exploratoire des disparités régionales dans l'Europe élargie", Région et développement, 21, pp. 65-92.

ERTUR, C., KOCH W. (2006), "Regional disparities in the European Union and the enlargement process : an exploratory spatial data analysis, 1995-2000", The Annals of Regional Science, 40, 4, pp. 723-765.

ERTUR C., LE GALLO J. \& BAUMONT C. (2006), "The European regional convergence process, 1980-1995 : Do spatial regimes and spatial dependence matter?", International Regional Science Review, 29, 1, pp. 3-34.

FAGGIAN A., MCCANN P. (2009), Higher education, graduate migration and regional dynamism in Great Britain. Working paper.

FRATESI U. (2016), 28. Impact assessment of EU Cohesion policy : theoretical and empirical issues. Handbook on Cohesion Policy in the EU, p. 443.

FUJITA M., KRUGMAN P. \& Venables A. J. (1999), The spatial economy, Cambridge MIT Press. GAGLIARDI L., PERCOCO M. (2017), “The impact of European Cohesion Policy in urban and rural regions", Regional Studies, 51, 6, pp. 857-868. 
GALLO J., ERTUR C. (2003), “Exploratory spatial data analysis of the distribution of regional per capita GDP in Europe, 1980-1995”, Papers in regional science, 82, 2, pp. 175-201.

GEARY R.C. (1954), "The contiguity ratio and statistical mapping”, The Incorporated Statistician 5, 3, pp. 115-146.

GEPPERT K., STEPHAN A. (2008), "Regional disparities in the European Union : Convergence and agglomeration”, Papers in Regional Science, 87, 2, pp. 193-217.

GETIS A. (2010), "Spatial filtering in a regression framework : examples using data on urban crime, regional inequality, and government expenditures", in Perspectives on spatial data analysis, Springer Berlin Heidelberg, pp. 191-202.

GETIS A., ORD J.K. (1992), “The analysis of spatial association by use of distance statistics”, Geographical analysis, 24, 3, pp. 189-206.

GLAESER E.L., LA PORTA R., LOPEZ-DE-SILANES F. \& SHLEIFER A. (2004), “Do institutions cause growth ?", Journal of economic Growth, 9, 3, pp. 271-303.

IAMMARINO S., RODRÍGUEZ-POSE A. \& STORPER M. (2017), Why regional development matters for Europe's economic future, Document de la Commission européenne.

KALDOR N. (1970), “The case for regional policies”, Scottish journal of political economy, 17, 3, pp. 337-348.

KELLY C., LAIRD J., COSTANTINI S., RICHARDS P., CARBAJO J. \& NELLTHORP J. (2015), "Ex post appraisal : What lessons can be learnt from EU cohesion funded transport projects ?", Transport Policy, 37, pp. 83-91.

KETTERER T.D., RODRÍGUEZ-POSE A. (2016), “Institutions vs.'first-nature'geography : What drives economic growth in Europe's regions?", Papers in Regional Science.

KOCH W. (2004), Effets de voisinage dans le modèle de Solow avec des externalités spatiales, Document de travail, Laboratoire d'économie et de gestion (LEG).

KRUGMAN P. (1991), "Increasing returns and economic geography”, Journal of political economy, 99, 3, pp. 483-499.

LE GALLO J. (2002), “Econométrie spatiale : l'autocorrélation spatiale dans les modèles de régression linéaire", Economie \& prévision, 4, pp. 139-157.

LE GALLO J., DALL'ERBA S. \& GUILLAIN R. (2011), “The local versus global dilemma of the effects of structural funds", Growth and Change, 42, 4, pp. 466-490.

LEONARDI R. (2005), Cohesion policy in the European Union : the building of Europe, Springer.

LESLIE T.F., KRONENFELD B.J. (2011), “The Colocation Quotient : A New Measure of Spatial Association Between Categorical Subsets of Points", Geographical Analysis, 43, 3, pp. 306-326.

LÓPEZ-BAZO E., VAYÁ E., MORA A. J. \& SURIÑACH J. (1999), "Regional economic dynamics and convergence in the European Union", The Annals of Regional Science, 33, 3, pp. 343-370.

LUCAS R.E. (1998), “On the mechanics of economic development”, Econometric Society Monographs, 29 , pp. 61-70.

MAYNOU L., SAEZ M., KYRIACOU A. \& BACARIA J. (2016), “The impact of structural and cohesion funds on Eurozone convergence, 1990-2010", Regional Studies, 50, 7, pp. 1127-1139. 
MESSNER S.F., ANSELIN L., BALLER R. D., HAWKINS D.F., DEANE G. \& TOLNAY S.E. (1999), “The spatial patterning of county homicide rates : an application of exploratory spatial data analysis", Journal of Quantitative criminology, 15, 4, pp. 423-450.

MOHL P. (2016), "Econometric evaluation of EU Cohesion Policy : a survey", in MOHL (ed.), Empirical Evidence on the Macroeconomic Effects of EU Cohesion Policy, Springer Gabler, Wiesbaden, pp. 7-35.

MOHL P., HAGEN T. (2010), "Do EU structural funds promote regional growth ? New evidence from various panel data approaches”, Regional Science and Urban Economics, 40, 5, pp. 353-365.

MOLLE W. (2007), European cohesion policy, Routledge.

MOLLE W. (2015), Cohesion and growth : the theory and practice of European policy making, Routledge.

MORAN P.A. (1950), “Notes on continuous stochastic phenomena”, Biometrika, 37, 1/2, pp. 17-23.

MYRDAL G. (1957), “The principle of circular and cumulative causation”, in GUNNAR MYRDAL, Rich Lands and Poor : The Road to World Prosperity, New York, Harper, pp. 11-22.

NISTOTSKAYA M., CHARRON N. \& LAPUENTE V. (2015), "The wealth of regions : quality of government and SMEs in 172 European regions", Environment and Planning C : Government and Policy, 33, 5, pp. 1125-1155.

OCDE (2009), Regions Matter : Economic Recovery, Innovation and Sustainable Growth, Paris, France

OLIVEAU S. (2010), “Autocorrélation spatiale : leçons du changement d'échelle”, L'Espace géographique, 39, 1, pp. 51-64.

PELLEGRINI G., TERRIBILE F., TAROLA O., MUCCIGROSSO T. \& BUSILLO F. (2013), "Measuring the effects of European Regional Policy on economic growth : A regression discontinuity approach", Papers in Regional Science, 92, 1, pp. 217-233.

PERCOCO M. (2017), "Impact of European Cohesion Policy on regional growth : does local economic structure matter ?", Regional Studies, 51, 6, pp. 833-843.

PERROUX F. (1955), “Trois outils d'analyse pour l'étude du sous-développement”, Cahiers de l'ISEA, 1.

QUAH D.T. (1996), “Empirics for economic growth and convergence”, European economic review, 40, 6, pp. 1353-1375.

RAMAJO J., MARQUEZ M. A., HEWINGS G. J. \& SALINAS M.M. (2008), "Spatial heterogeneity and interregional spillovers in the European Union : Do cohesion policies encourage convergence across regions ?", European Economic Review, 52, 3, pp. 551-567.

RODRÍGUEZ-POSE A., DI CATALDO M. (2014), "Quality of government and innovative performance in the regions of Europe", Journal of Economic Geography, 15, 4, pp. 673-706.

RODRÍGUEZ-POSE A., FRATESI U. (2004), "Between development and social policies : the impact of European Structural Funds in Objective 1 regions”, Regional Studies, 38, 1, pp. 97-113.

RODRÍGUEZ-POSE A., GARCILAZO E. (2015), “Quality of government and the returns of investment : Examining the impact of cohesion expenditure in European regions", Regional Studies, 49, 8, pp. 1274-1290.

RODRÍGUEZ-POSE A., KETTERER T. (2018), Institutional change and the development of lagging regions in Europe (No. 1808), Universidade de Vigo, GEN-Governance and Economics research Network. 
RODRÍGUEZ-POSE A. (2018), “The revenge of the places that don't matter (and what to do about it)", Cambridge Journal of Regions, Economy and Society, 11, 1, pp. 189-209.

ROMER P.M. (1986), “Increasing returns and long-run growth”, Journal of political economy, 94, 5, 1002-1037.

ROSIK P., STĘPNIAK M. \& KOMORNICKI T. (2015), “The decade of the big push to roads in Poland : Impact on improvement in accessibility and territorial cohesion from a policy perspective", Transport Policy, 37, 134-146.

SHI H., ZHANG L. \& LIU J. (2006), “A new spatial-attribute weighting function for geographically weighted regression”, Canadian journal of forest research, 36, 4, pp. 996-1005.

SOLOW R.M. (1956), “A contribution to the theory of economic growth", The quarterly journal of economics, 70, 1, pp. 65-94.

SONN J.W., STORPER M. (2008), “The increasing importance of geographical proximity in technological innovation : an analysis of U.S. patent citations, 1975-1997”, Environment and Planning A, 5, 40, pp. 1020-1039

SOTIRIOU A., TSIAPA M. (2015), "The asymmetric influence of structural funds on regional growth in Greece", Environment and Planning C : Government and Policy, 33, 4, pp. 863-881.

STORPER M., VENABLES A. (2004), "Buzz : face-to-face contact and the urban economy”, Journal of Economic Geography, 4, 4, pp. 351-370

SWAN T.W. (1956), "Economic growth and capital accumulation", Economic record, 32, 2, pp. 334-361.

VICKERMAN R., SPIEKERMANN K. \& WEGENER M. (1999), “Accessibility and economic development in Europe", Regional studies, 33, 1, pp. 1-15.

WARD T., WOLLEB E. (2006), Ex Post Evaluation of Cohesion Policy Programmes 2000-2006 financed by the European Regional Development Fund in Objective 1 and 2 Regions : Synthesis Report, March 2010, $176 \mathrm{p}$.

WARTENBERG D. (1985), “Multivariate spatial correlation : a method for exploratory geographical analysis”, Geographical Analysis, 17, 4, pp. 263-283.

WILLIAMSON J.G. (1965), "Regional inequality and the process of national development : a description of the patterns", Economic development and cultural change, 13, 4, Part 2, pp. 1-84.

\section{NOTES}

1. Pour une revue de littérature récente, nous renvoyons par exemple aux articles de Fratesi, 2016 ; Dall'Erba et Fang, 2017. 


\section{RÉSUMÉS}

La Politique de Cohésion de l'UE a fêté ses vingt années d'existence en 2009. La mise sur pied de cette politique part du constat que les forces du marché ne sont pas nécessairement suffisantes pour réduire significativement les inégalités territoriales. L'UE a alors construit cet outil de solidarité financière entre États membres avec l'objectif d'améliorer la compétitivité des régions défavorisées et de corriger les déséquilibres régionaux. Pourtant, la littérature sur la question de l'efficacité des Fonds européens n'est pas unanime. Aussi, nous proposons d'analyser la Politique de Cohésion et son rôle dans la croissance régionale au travers d'une méthode développée en analyse spatiale, à savoir les BiLISA. Les résultats montrent que l'influence de la Politique de Cohésion est différenciée en fonction des régions de l'UE et met en évidence des effets de concentrations spatiales. Les variations spatiales de l'influence des Fonds européens sur la croissance économique des régions appellent des inflexions dans la Politique de Cohésion, en particulier en faveur d'une politique davantage territorialisée.

EU Cohesion Policy celebrated its twenty years of existence in 2009. The establishment of this policy stems for the observation that market forces may not be sufficient to significantly reduce regional inequalities. The EU therefore built this financial solidarity tool between member States with the aim of improving the competitiveness of disadvantaged regions and correcting regional imbalances. Yet, literature (particularly in spatial econometrics) on the question of the effectiveness of European funds is not unanimous. Hence, we suggest to analyse the cohesion policy and its role in regional growth through a method developed in spatial analysis, namely BiLISA. The results show that the effects of the cohesion policy are differentiated according to EU regions and highlight spatial dependence effects. Spatial variations of the influence of European funds on the economic growth of regions ask for inflections in the Cohesion policy, especially in favor of a more territorialized policy.

\section{INDEX}

Mots-clés : Politique de Cohésion, croissance régionale, autocorrélation spatiale, Union européenne

Keywords : cohesion policy, regional growth, spatial autocorrelation, European union

\section{AUTEUR}

\section{SÉBASTIEN BOURDIN}

Professeur Associé en Développement Durable et Territorial

Département Economie des Territoires et Développement Durable, Ecole de management de Normandie, sbourdin@em-normandie.fr 